\title{
Effect of Conditioned Medium from Human Umbilical Cord-Derived Mesenchymal Stromal Cells on Rejuvenation of Nucleus Pulposus Derived Stem/Progenitor Cells from Degenerated Intervertebral Disc
}

\author{
Xiaoli Zeng ${ }^{1, *}$, Jinhua Lin ${ }^{1, *}$, Hao $\mathrm{Wu}^{2}$, Jiayue $\mathrm{Yu}^{1}$, Mei $\mathrm{Tu}^{3}$, Lek Hang Cheang ${ }^{4}$, Jiaqing Zhang $^{1}$ \\ ${ }^{1}$ Department of Biochemistry and Molecular Biology, School of Preclinical Medicine, Finan University, Guangzhou, China \\ ${ }^{2}$ Department of Orthopedic Surgery, The First Affiliated Hospital, Finan University, Guangzhou, China \\ ${ }^{3}$ Department of Materials Science and Engineering, Finan University, Guangzhou, China \\ ${ }^{4}$ Department of Orthopedic Surgery, Centro Hospitalar Conde de Sao Fanuario, Macao, China
}

Background and Objectives: Mesenchymal stromal cells (MSCs)-based treatment for degeneration of intervertebral disc (IVD) has been proposed recently. We here addressed whether MSC secreted factors can rejuvenate nucleus pulposus-derived stem/progenitor cells from degenerated disc (D-NPSCs) in vitro.

Methods and Results: We analyzed the expression of MSCs and NP cell specific surface markers, pluripotency related genes, multilineage potential and cell proliferative capacity of D-NPSCs upon incubation with the conditioned medium which was collected from the umbilical cord derived MSCs (UCMSCs). Our results indicated that the conditioned medium restore the stemness of D-NPSCs by up-regulating the expression level of CD29 and CD105, pluripotency related genes OCT4 and Nanog, and NP progenitor marker Tie2. The increased stemness was accompanied by promoted cell proliferative capacity and improved osteogenic and chondrogenic differentiation potential.

Conclusions: Our findings suggested that the UCMSCs derived conditioned medium might be used to rejuvenate the degenerated NP stem/progenitor cells.

Keywords: Conditioned medium, Mesenchymal stromal cells, Nucleus pulposus derived stem/progenitor cells, Intervertebral disc degeneration

Received: February 11, 2020, Revised: April 8, 2020,

Accepted: April 14, 2020, Published online: June 30, 2020

Correspondence to Jiaqing Zhang

Department of Biochemistry and Molecular Biology, School of Preclinical Medicine, Jinan University, Huangpu Road 601, Guangzhou 510632, China

Tel: +86-20-85220256, Fax: +86-20-85223271

E-mail: zhangjiaqing@jnu.edu.cn

${ }^{*}$ These authors contributed equally to this work.

(c) This is an open-access article distributed under the terms of the Creative Commons Attribution Non-Commercial License (http://creativecommons.org/ licenses/by-nc/4.0/), which permits unrestricted non-commercial use, distribution, and reproduction in any medium, provided the original work is properly cited.

Copyright (c) 2020 by the Korean Society for Stem Cell Research

\section{Introduction}

Intervertebral disc (IVD) degeneration and consequent low-back pain have been the leading cause of disability especially in people aged less than 45 years old $(1,2)$, which presents a costly burden to the healthcare system. Conventional treatments for IVD degeneration are limited, since conservative or surgical therapies do not restore IVD tissue properties. Stem-cell based therapies that focus on rejuvenating and functionalizing tissue are becoming a promising strategy for IVD degeneration (3). Exogenous mesenchymal stem/stromal cells (MSCs), especially umbilical cord derived MSCs (UCMSCs), are currently the 
most popular graft cells for IVD regeneration because UCMSCs provide a nearly unlimited cell source with self-renewal capability and multilineage differentiation potential $(4,5)$. However, IVD microenvironment is characterized by high osmolarity, limited nutrition, acidic $\mathrm{pH}$, and low oxygen tension $(6,7)$. Some studies have reported that exogenous stem cells are not able to survive well under IVD harsh microenvironment, as it negatively influences the biological and metabolic vitality of stem cells (8, 9). The IVD is composed of an inner nucleus pulposus (NP) surrounded by the annulus fibrosus (AF) and thin hyaline cartilaginous end-plates (EP) between the IVD and the adjacent vertebral bodies. Increasing evidence showed that cells isolated from the various compartments of human IVD, such as NP, AF, and EP expressed most of the phenotype markers that define MSCs, thus supporting the existence of resident stem/progenitor cells within IVD $(10,11)$. As seed cell candidate, the endogenous stem cells inside the IVD, especially nucleus pulposus-derived stem/progenitor cells, exhibit better adaptability to the harsh IVD microenvironment than the adipose-derived MSCs in tolerating hypoxic (12), hypertonic (13), and acidic culture conditions (14) after being transplanted into IVD. Therefore, the endogenous stem cells inside the IVD had been regarded as attractive seed cells and might perform better than exogenous MSCs for IVD regeneration (15). Nonetheless, the number of stem/progenitor cells that can be harvested and expanded from nucleus pulposus (NP) tissues in vitro are insufficient to meet requirements for successful treatment (16). In addition, culturing primary NP stem/progenitor cells for disc repair is challenging due to their limited expansion capability $(17,18)$. These issues have motivated the exploration of rejuvenating regenerative capacity of NP derived stem/progenitor cells from degenerated IVD.

It has been reported that MSCs exhibit a number of trophic functions to enhance tissue regeneration (19). Recent studies have suggested that MSCs can promote the viability of NP cells in animal models and in preclinical studies $(20,21)$. Sun et al. (22) noted that adipose-derived stem cells protect compressive load induced NP cell death and degradation by inhibition of activated caspase- 9 and -3 activity in the co-culture system. Yamamoto et al. (23) observed that bone marrow derived MSCs may improve the viability of NP Cells in vitro. Results from these studies indicated that the mechanism underlying MSCs efficacy depends mostly on their paracrine activity.

However, researches reporting the ability of MSC secretome to promote the functionality of NP cells are just emerging. Whether the trophic factors secreted by MSCs can ameliorate the biological function of NP derived stem/ progenitor cells from degenerated disc (D-NPSCs) is not fully understood, and continuous laboratory investigations are indispensable to explore the possibility of employing trophic factors secreted by MSCs to promote the regenerative capacity of D-NPSCs and further develop a stem cellbased treatment for degenerated IVD.

Despite the presence of many different types of stem cells, UCMSCs have been recently considered more potent as cell source than bone marrow MSCs or adipose-derived MSCs since they proliferate/differentiate more efficiently and have a noninvasive harvesting procedure as well as abundant cell sources (24). Thus, in this study, we collected conditional medium from human UCMSCs (UCMSCs-CM) to treat D-NPSCs, which were isolated from degenerated NP tissues obtained from patients who underwent micro endoscopic discectomy for degenerative spine diseases. We found that UCMSCs-CM exposure restored biological activities of D-NPSCs detected by examining the pluripotent markers, proliferative capacity, multi-lineage differentiation potential, and the expression of NP-specific cell surface markers. Our finding will lay a foundation for the development of optimal therapeutic strategy for IVD degeneration.

\section{Materials and Methods}

\section{Ethics approval}

This study was approved by the medical ethics committee of Jinan University, Guangzhou, China. All procedures performed for the present work were approved specific informed consent was obtained in all cases.

\section{Sample collection}

Mildly as well as severely degenerated NP tissue samples were obtained from patients $(n=8)$ who underwent micro endoscopic discectomy for degenerative spine diseases. The details of all samples and patients are shown in Table S1 of the Supplementary File.

Umbilical cord were obtained from newborns $(n=5)$ who exhibited no complications throughout pregnancy and were delivered full-term by Caesarean section.

\section{Isolation of cells from NP tissues or umbilical cord \\ Isolation of cells from NP tissues: The disc tissue} samples were collected under sterile conditions immediately following surgery, washed with phosphate-buffered saline (PBS, $\mathrm{pH}=7.2$ ). The NP tissue were obtained by removing anulus fibrosus tissue under stereoscopic microscope. The explant culture method was employed to iso- 
late NPSCs from NP tissue as previously described (25). In brief, NP tissues were finely minced into small fragments of approximately $1 \mathrm{~mm}^{3}$, and incubated at $37^{\circ} \mathrm{C}$ in a humidified atmosphere containing $5 \% \mathrm{CO}_{2}$ without culture medium for two hours to allow the tissue pieces to attach. We used Dulbecco Modified Eagle Medium (DMEM)/F-12 (Gibco, USA) supplemented with 10\% fetal bovine serum (FBS) (Gibco, USA), 1\% L-glutamine, and $1 \%$ penicillin-streptomycin (Invitrogen, USA) as complete culture medium, which was added to the tissue culture dishes and incubated for an additional 15 18 days. The medium was changed every two days. The pieces were removed when the cells reached $80 \%$ confluency, and the cells were passaged by a 2 -min treatment with $0.25 \%$ trypsin and $0.02 \%$ EDTA at $37^{\circ} \mathrm{C}$. Cells at Passage $2 \sim 4$ cells were used in the experiment.

Isolation of MSCs from human umbilical cord: UCMSCs were isolated and cultured as described previously (26). Briefly, umbilical cord tissue was washed twice with PBS. A $10 \mathrm{~cm}$ umbilical cord was cut into small pieces of about $1 \mathrm{~cm}$ in length. The pieces were washed thoroughly to remove blood and blood clots, the Wharton's jelly was separated and dissected into small pieces of approximate $1 \sim 2 \mathrm{~mm}^{3}$ pieces, then plated in the complete culture medium and cultured for 12 days. The medium was changed every third day. At 80\% 90\% confluence, UCMSCs were detached and passaged. Cells at Passage 3 $\sim 6$ were used for all experiments.

Collection of conditioned medium from UCMSCs cultures: UCMSCs were cultured and expanded in the complete culture medium. At passage $3 \sim 6$, a total of $1 \times 10^{6}$ cells were seeded into $100 \mathrm{~mm}^{2}$ cell culture dishes. When the cells reached $60 \%$ confluence, they were washed with PBS and then cultured in serum-free complete culture medium for an additional $24 \mathrm{~h}$. The cell supernatant was collected and filtered with 0.22 micron filter to remove living cells, dead cells and cellular debris as conditioned medium, stored at $-80^{\circ} \mathrm{C}$ until use. When used, the conditioned medium would be mixed with DMEM/F12 at the concentration of $40 \%$, which was further supplemented with $10 \%$ FBS, 1\% L-glutamine and $1 \%$ penicillin-streptomycin, as UCMSCs-CM.

To determine the optimal percentage of the conditioned medium, we performed the pre-experiment to evaluate the effects of $20 \%, 40 \%$ and $80 \%$ of the conditioned medium on the proliferative capacity of D-NPSCs by using CCK 8 method. The result showed that the OD values of $40 \%$ were the highest compared with other groups (data shown in Fig. S1 of the Supplementary File).

D-NPSCs in passage $2 \sim 4$ were plated at $5 \times 10^{4}$ cells/well in 24-well culture plates, then treated with UCMSCs-CM, as experimental group (CM group), the untreated DNPSCs was taken as negative control (D-NPSCs group) and UCMSCs was acted as positive control (UCMSCs group). The media used in D-NPSCs and UCMSCs group were complete culture medium, which contained DMEM/ F12 supplemented with 10\% FBS, 1\% L-glutamine and $1 \%$ penicillin-streptomycin.

\section{Flow cytometry assay}

The expression of specific surface markers: The characteristics of the cells from D-NPSCs, CM and UCMSCs group were investigated further by analyzing the cell surface markers with flow cytometry. Cells at passage 2 4 were harvested and washed with PBS after $72 \mathrm{~h}$ treatment of UCMSCs-CM, and every $1 \times 10^{6}$ cells were resuspended in $100 \mu 1$ cold PBS. Fluorochrome-coupled anti-mouse monoclonal antibodies (including CD29-FITC, CD44-PE, CD73-PE, CD90-PC5, CD105-PE, CD11b-PE, CD14-FITC, CD24-FITC, CD34-PE, CD45-PC5, CD45PerCP and HLA-DR-FITC) were added to the corresponding tubes, and were incubated with the cell suspension at $4{ }^{\circ} \mathrm{C}$ for $30 \mathrm{~min}$ in the dark. Isotype control antibodies served as controls. Finally, labeled cells were washed three times with PBS and surface markers expression was detected using flow cytometry (BD Biosciences, USA). The percentage of positively stained cells was calculated relative to the isotype control.

Cell cycle analysis: Cells from D-NPSCs, CM and UCMSCs group were harvested after $72 \mathrm{~h}$ treatment of UCMSCs-CM, washed twice with cold PBS, and fixed in $75 \%$ pre-chilled ethanol for over 12 hours at $4{ }^{\circ} \mathrm{C}$. The fixed cells were washed twice with ice-cold PBS, then stained with propidium iodide (PI, $500 \mathrm{ml} / 10^{6}$ cells) at room temperature for $30 \mathrm{~min}$ in the dark. The intensity of fluorescence was determined by flow cytometry, and the percentages of cells in G0/G1, G2/M and S phases were analyzed using BD CellQuest software. The experiment was repeated three times to ensure reproducibility.

Apoptosis analysis: Cell apoptotic fraction was detected by staining with FITC-conjugated annexin-V and PI using the Annexin-V/PI Apoptosis Detection kit. According to the manufacturer's instructions, all cells were harvested after $72 \mathrm{~h}$ treatment of UCMSCs-CM, washed twice with PBS, and resuspended in $300 \mu 1$ of binding buffer. $5 \mu 1$ Annexin V-FITC solution was added to the cells and incubated for $30 \mathrm{~min}$ at $4^{\circ} \mathrm{C}$ in the dark, which was followed by further incubation with $5 \mu \mathrm{l}$ PI for $5 \mathrm{~min}$. The percentage of apoptotic cells were analyzed immediately by flow cytometry, and the experiment was 
repeated three times independently.

\section{Cell viability and proliferation analysis}

Growth curve: Growth curve was plotted to evaluate growth kinetics of the cells after 13 days incubation of UCMSCs-CM. Cells for each group were seeded at a density of $3 \times 10^{4}$ cells/well in 24 -well plates. The cells were harvested by trypsinization from each well, and a duplicate was collected and counted with a hemocytometer count at day 1 , day 3 , day 5 , day 7 , day 9, day 11 and day 13. The growth curve was plotted with the cell culture time as the horizontal axis and the number of cells as the vertical axis. The results were analyzed by GraphPad Prism software.

Cell viability: Cell viability was determined using the Cell Counting Kit 8 (CCK-8, Dojindo Laboratories, Japan). A total of $3 \times 10^{4}$ cells/well were seeded in 24-well plates, CCK-8 solution was added at day $1,3,5$ and 7 , and incubated for a further $2.5 \mathrm{~h}$. The absorbance of the supernatant was measured with a micro plate reader (Bio-Rad, USA) at wavelength of $450 \mathrm{~nm}$. The CCK8 assays were performed in triplicate.

EdU incorporation assay: The EdU (5-ethynyl-2'-deoxyuridine) proliferation assay was performed to further explore the role of UCMSCs-CM using Cell-Light ${ }^{\mathrm{TM}} \mathrm{EdU}$ Apollo 567 In Vitro Kit (Ribobio, China) following the manufacture's recommendation. In brief, cells were seeded into 96-well plates at $5 \times 10^{3}$ cells/well, and cultured for $72 \mathrm{~h}$. Then $100 \mu \mathrm{l}$ medium containing $50 \mu \mathrm{M}$ EdU was added into each well and cells were incubated for $2 \mathrm{~h}$ at $37^{\circ} \mathrm{C}$, fixed with $4 \%$ par formaldehyde (PFA), then stained with Hoechst 33342 and Apollo reaction cocktail. All images were processed using Image $J$ software and the proportion of EdU incorporated cells was calculated. Three independent experiments were performed for quantification.

\section{Multilineage differentiation}

Osteogenic and adipogenic differentiation of sub confluent cells was induced by induction media for 21 days as previously described by Richardson et al. (27) after 72 $\mathrm{h}$ treatment of UCMSCs-CM. For chondrogenesis, $3.5 \times 10^{6}$ cells were treated with UCMSCs-CM for $72 \mathrm{~h}$, then centrifuged to form a three-dimensional aggregate in a $15 \mathrm{ml}$ conical tube, and incubated with chondrogenic media for 21 days. The induced medium was replaced every $2 \sim 3$ days. For Alizarin Red S (ARS) staining, cells were fixed in 4\% PFA for 10 minutes and stained for 5 10 min with $40 \mathrm{mM}$ ARS solution. Oil red $\mathrm{O}$ solution was used to stain intracellular lipid vacuoles for 5 10 min after fixation with PFA. To identify chondrogenic differentiation, the cell pellets were stained with Alcian blue, which were fixed in PFA, then frozen in OCT freezing medium (Sakura Finetek), sectioned into $5 \mu \mathrm{m}$-thick slices. The results were photographed under phase contrast microscope, and all images were processed using Image $\mathrm{J}$ software, the proportion of area positively stained was calculated. The experiment was repeated 3 times independently.

\section{The expression of marker genes detected with quantitative real-time polymerase chain reaction (qRT-PCR)}

Quantitative real-time polymerase chain reaction (qRTPCR) was used to determine relative gene expression. The expression of NP-specific markers genes Aggrecan (ACAN), COL2A1, SOX9, Tie2, and CD24, and pluripotent stem cell-related genes (OCT4 and Nanog) were evaluated after $72 \mathrm{~h}$ incubation of UCMSCs-CM. All primers were designed according to the sequences in GenBank using software prime 5.0. Sequences of primers were shown in Table 1. Cells were harvested, and total RNA was extracted using Trizol reagent (Invitrogen, USA), and quantified by a Nanodrop 2000 spectrophotometer (Thermo Fisher, USA). RNA (1 $\mu \mathrm{g})$ was used for cDNA synthesis using a Cell to cDNA Kit. Real-time PCR was then per-

Table 1. Primers used for this study

\begin{tabular}{lll}
\hline Gene name & \multicolumn{1}{c}{ Forward primer $\left(5^{\prime}\right.$ to $\left.3^{\prime}\right)$} & \multicolumn{1}{c}{ Reverse primer $\left(5^{\prime}\right.$ to $\left.3^{\prime}\right)$} \\
\hline SOX9 & AGCGAACGCACATCAAGAC & CTGTAGGCGATCTGTTGGGG \\
ACAN & TCGAGGACAGCGAGGCC & TCGAGGGTGTAGCGTGTAGAGA \\
COL2A1 & GGCAATAGCAGGTTCACGTACA & CGATAACAGTCTTGCCCCACTT \\
CD24 & GCACTGCTCCTACCCACGCAGATTT & GCCTTGGTGGTGGCATTAGTTGGAT \\
Tie2 & AATCACTATGAGGCTTGGCAACAT & GCGTCTCACAGGTCCAGGAT \\
NANOG & GATTTGGGGCCTGAAGAAA & CAGATCCATGGAGGAAGGAA \\
OCT4 & GAGAAGGAGAAGCTGGAGCA & AATAGAACCCCAGGGTGAG \\
GAPDH & CAGCGACACCCACTCCTC & TGAGGTCCACCACCCTGT \\
\hline
\end{tabular}


formed in a final volume of $20 \mu 1$ with $2 \times$ SYBR Green qRT-PCR Master Mix. Each reaction was performed in triplicate. The housekeeping gene $G A P D H$ was used for endogenous control. The $2^{-\triangle \triangle \mathrm{Ct}}$ method was used to describe the relative expression levels of the gene.

\section{Statistical analysis}

The data are presented as the mean \pm SD. One-way analysis of variance (ANOVA) was conducted to analyze the differences among different groups. $\mathrm{p}<0.05$ was considered statistically significant.

\section{Results}

To investigate effects of the conditioned medium de- rived from UCMSCs on regenerative capacity of NP resident stem/progenitor cells from degenerated IVD, DNPSCs were treated with the UCMSCs-CM at $40 \%$ of the conditioned medium and compared with untreated DNPSCs and UCMSCs in term of immunophenotype, proliferation, multi-lineage differentiation abilities, and the expression levels of NP related markers.

\section{UCMSCs-CM enhanced expression of MSC surface markers in D-NPSCs}

Flow cytometry results showed that UCMSCs were highly positive for CD29, CD44, CD73, CD90 and CD105 at rates greater than $95 \%$, and negative for CD11b, CD14, CD34, CD45 and HLA-DR (Fig. 1), which fulfilled the international Society for Cell Therapy (ISCT) require-
A

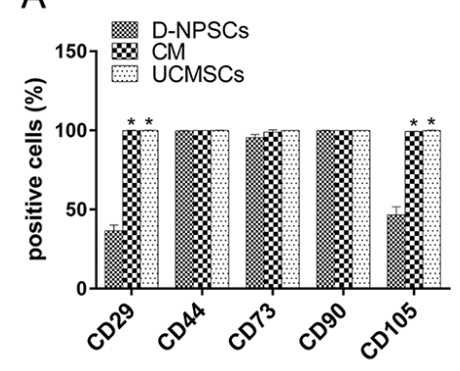

B

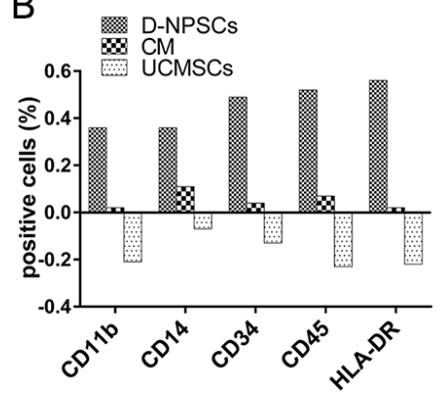

C

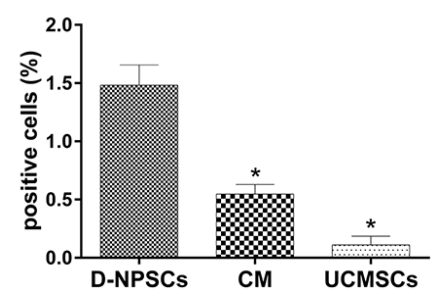

Fig. 1. The MSC and NP cell surface markers expression were analysis by flow cytometry after $72 \mathrm{~h}$ treatment with UCMSCs-CM. (A) MSC positive surface markers, (B) MSC negative surface markers, (C) NP cell surface marker CD24. The data are presented as the means \pm SD, $\mathrm{n}=3 .{ }^{*} \mathrm{p}<0.05$, compare with D-NPSCs group. UCMSCs were positive for CD29, CD44, CD73, CD90 and CD105 at rates greater than $95 \%$. For D-NPSCs, the expression of CD29, and CD105 showed interindividual variability but all at rates lower than $95 \%$, however, CD29 and CD105 in CM group were at rates greater than 95\%, and the CM group revealed a very similar expression pattern of surface markers with UCMSCs group; Cells in all three groups were negative for the hematopoietic stem cell surface markers CD11b, CD14, CD34, CD45 and HLA-DR. Cells in all three groups expressed CD24 at low rates, while the rate in D-NPSCs significantly higher than that in both CM group and UCMSCs group.
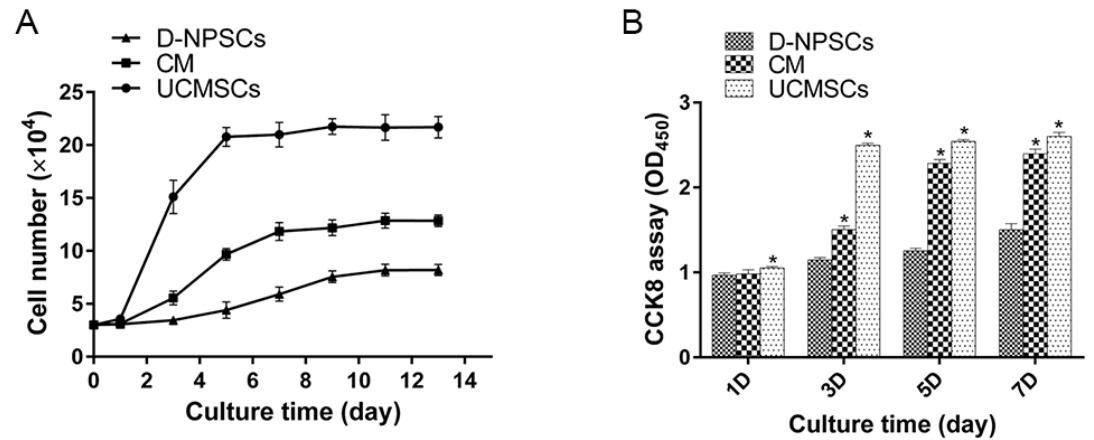

Fig. 2. Proliferation capability analysis of cells in the three groups. (A) Growth curves, (B) Cell viability was analyzed by cell counting kit-8 on the $1^{\text {st }}, 3^{\text {rd }}, 5^{\text {th }}$, and $7^{\text {th }}$ day. The data are expressed as mean $\pm S D, n=3,{ }^{*} p<0.01$ vs. D-NPSCs. (A) Both UCMSCs and CM group were accelerated rapidly during days $3 \sim 5$ (logarithmic phase), and slowed down thereafter (stationary phase), while D-NPSCs in the days 3 , cells proliferated slowly and then entered the logarithmic growth phase, which continued for $5 \sim 6$ days, and reached cell growth plateau in 9 13 days. (B) Cells from CM group exhibited a greatly increased OD value compared with D-NPSCs group at day 3, 5 and 7. 
ments for MSC definition; The D-NPSCs before UCMSCs-CM treatment, although expression of CD73, CD44 and CD90 were at rates greater than 95\% and expression of CD11b, CD14, CD34, CD45 and HLA-DR were negative, expression of CD29 and CD105 showed interindividual variability and all at rates lower than 95\%. However, CD29 and CD105 were significantly up-regulated at rates greater than $95 \%$ after $72 \mathrm{~h}$ UCMSCs-CM treatment (Fig. 1, p <0.05), therefore CM group exhibited a very similar expression pattern of surface markers with UCMSCs group.

\section{UCMSCs-CM promote proliferation and viability in D-NPSCs}

Growth curve: According to the growth curve, all cells proliferation was slow during the first day of culture (latent phase) as shown in Fig. 2A. Both UCMSCs and CM groups were accelerated rapidly during day 3 5 (logarithmic phase), and slowed down thereafter (stationary phase), while D-NPSCs proliferated still slowly on day 3, and then entered the logarithmic growth phase at day 5 , which continued for 5 6 days, and reached cell growth plateau at day $10 \sim 13$. These results demonstrated that the growth ability of cells in CM group was markedly higher than that in D-NPSCs group.

Cell viability analyzed by CCK8 method: The viability of D-NPSCs and UCMSCs was assessed with CCK8 method as shown in Fig. 2B. The OD values of cells from both CM group and UCMSCs at day 3, 5 and 7 were significantly higher than D-NPSCs, which was consistent with the results of growth curves. The CM group reached to a similar OD value with UCMSCs group at day 5, 7 .

EdU analysis: The results showed that cells in CM group had markedly higher proportion of EdU incorporated cell than D-NPSCs group after $72 \mathrm{~h}$ UCMSCsCM treatment (Fig. 3, p<0.01), although lower than UCMSCs group, which suggested that UCMSCs-CM promoted the DNA replication and cell growth in D-NPSCs.

Cell cycle and apoptosis analysis: Cell cycle were assessed using flow cytometry, the percentage of cells in the G1/G0, G2/M and S phases of cell cycle was then analyzed at $72 \mathrm{~h}$ following UCMSCs-CM treatment. The results revealed that cells in the CM group had significantly higher percentages of cells in the $\mathrm{S}$ phases and lower percentages

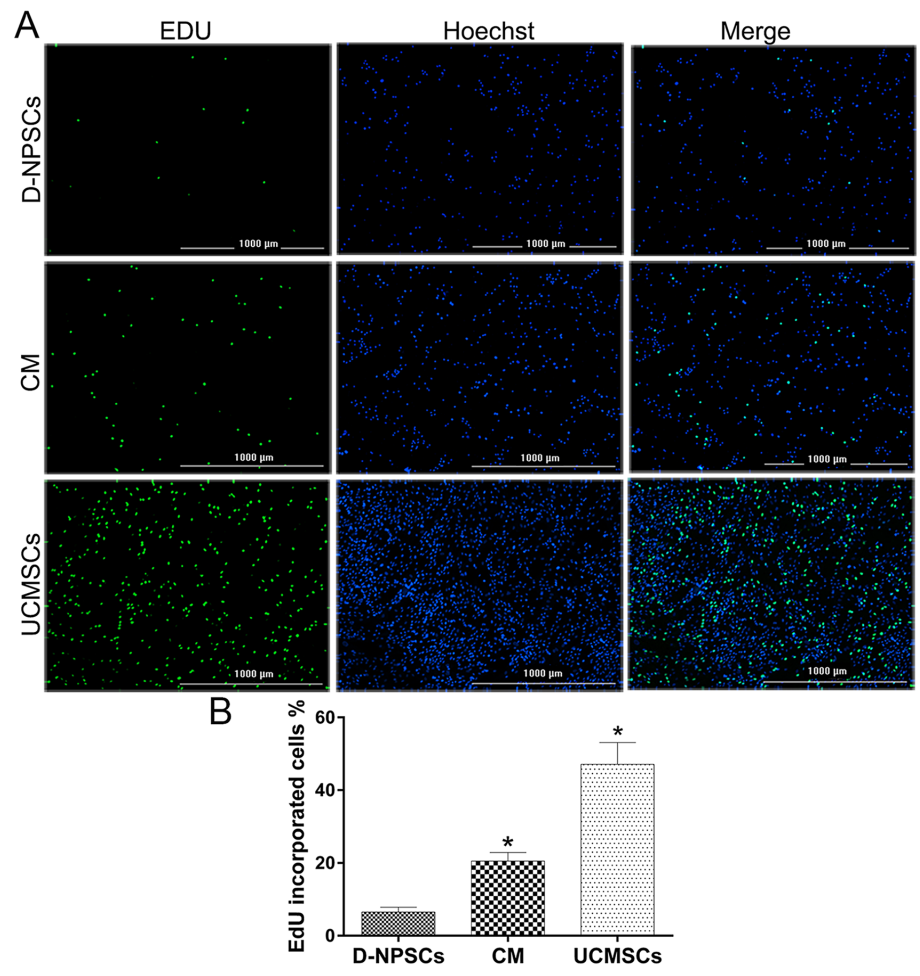

Fig. 3. EdU proliferation assay after $72 \mathrm{~h}$ treatment with UCMSCs-CM. (A) EdU incorporated cells in the three groups. (B) Comparative analysis of the percentage of EdU incorporated cells in the three groups. Scale bar $=1000 \mu \mathrm{m}$ (magnification $40 \times$ ). The data are presented as the means $\pm S D, n=3 .{ }^{*} p<0.01$, compare with D-NPSCs group. (A) Cells in D-NPSCs group had markedly lower proportion of EdU incorporated cell than CM group and UCMSCs group. (B) Further quantitative analysis also revealed that the percentage of EdU incorporated cell was significantly lower in D-NPSCs than that in CM group and UCMSCs group. 

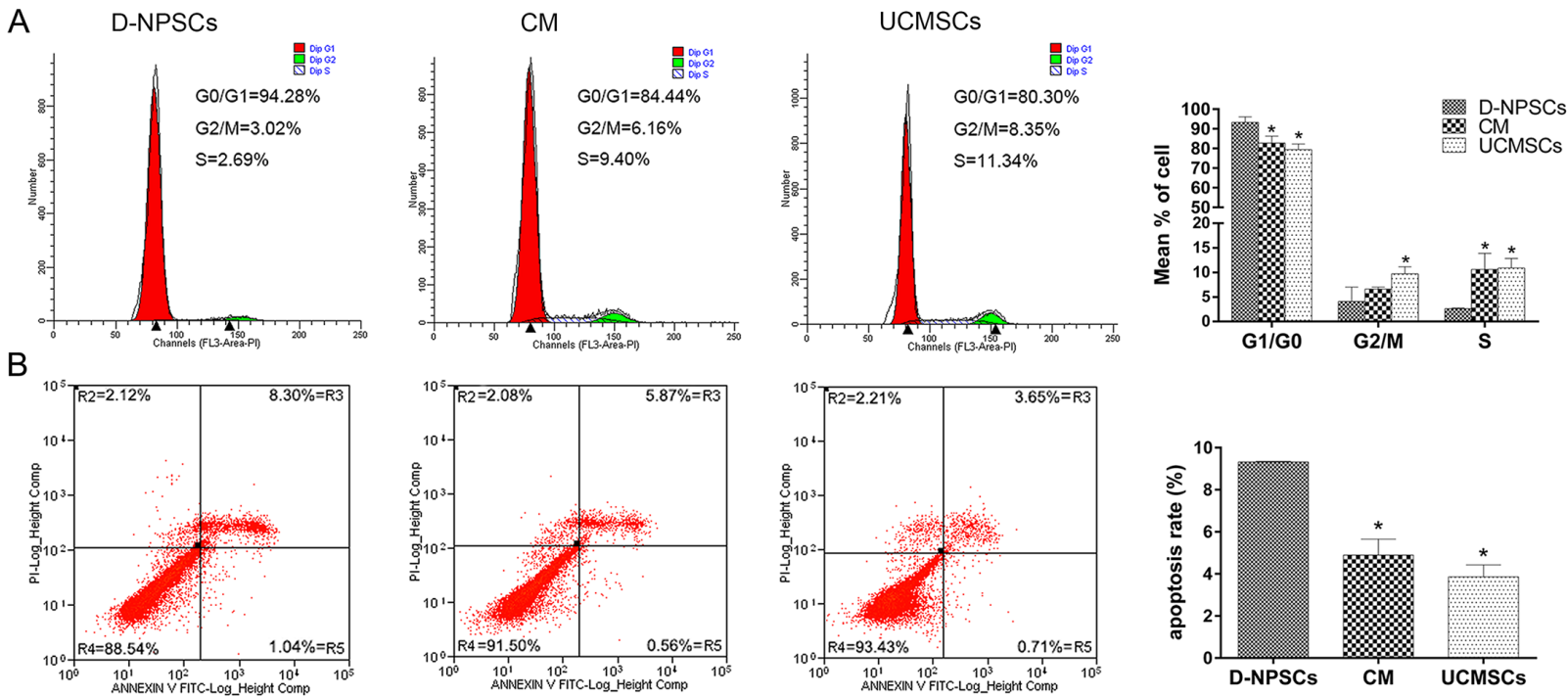

Fig. 4. The cell cycles (A) and apoptosis (B) were assessed after $72 \mathrm{~h}$ treatment with UCMSCs-CM by flow cytometry. The data are expressed as means $\pm S D, n=3,{ }^{*} p<0.05$ vs. D-NPSCs group. CM group had significantly higher percentages of cells in the $S$ phases and lower percentages of cells in the G1/G0 phase than D-NPSCs group, and showed a similarity with UCMSCs group (A). The cell apoptosis rate in CM group was significantly decreased compared with D-NPSCs group, and tended to be higher compared with UCMSCs group (B). Data are presented as the means $\pm \mathrm{SD}, \mathrm{n}=3$. ${ }^{*} \mathrm{p}<0.05$, compare with D-NPSCs group.

of cells in the G1/G0 phase than that in the D-NPSCs group (Fig. 4A, $\mathrm{p}<0.05$ ), which is similar to cells in the UCMSCs group. The frequency of cell apoptosis in the $\mathrm{CM}$ group was significantly decreased when compared with the D-NPSCs group after $72 \mathrm{~h}$ UCMSCs-CM treatment (Fig. 4B, p <0.05), and tended to be higher compared with the UCMSCs group.

Collectively, the proliferation and viability of cells in CM group were greatly higher than that of D-NPSCs group, indicated that UCMSCs-CM promoted stem/progenitor cell growth from degenerated nucleus pulposus by slowing down the process of cell apoptosis and driving more cells into the DNA synthesis phase.

\section{Multilineage differentiation potential analysis}

Multilineage differentiation potential were analysised when the cells were incubated for 21 days in adipogenic, osteogenic and chondrogenic media following UCMSCsCM treatment.

Osteogenic Differentiation D-NPSCs exhibited few calcium deposition stained by ARS as observed in Fig. 5A, whereas the cells from the $\mathrm{CM}$ group displayed larger and more intensely stained mineralized nodules $(p<0.01)$ though it presented less intense staining than UCMSCs.

Adipogenic Differentiation Oil red O was used to stain lipid-rich vacuoles to analyze for adipogenesis. Cells from all the three groups showed adipogenic differentiation.
However, there were no significant difference in positive staining area between the CM and D-NPSCs groups (Fig. $5 \mathrm{~B}$ ), and cells from both groups appeared to form less fat drops than UCMSCs.

Chondrogenic Differentiation Cells from CM group produced more intensely stained extracellular matrix than D-NPSCs group (Fig. 5C, p <0.01), but a similar staining intensity compared with UCMSCs group.

These data indicated that UCMSCs-CM improved the osteogenic and chondrogenic potential of D-NPSCs.

\section{UCMSCs-CM altered the expression level of NP related markers}

Results in Fig. 6 showed that the expression levels of NP cell phenotypic markers, SOX9, COL2A1 and ACAN in $\mathrm{CM}$ group were significantly lower than those in D-NPSCs group after $72 \mathrm{~h}$ UCMSCs-CM treatment (Fig. $6, p<0.01)$. Meanwhile, the mRNA expression of Tie2, a NP progenitor cell specific marker, and NP cell marker CD24, were also evaluated. The results showed that Tie2 expression was significantly up-regulated, while CD24 was significantly down-regulated in CM group compared with D-NPSCs group (Fig. 6, p $<0.01$ ).

Additionally, we had chosen CD24 for flow cytometry analysis, found that CD24 in CM group had a significantly lower positive rate than that in D-NPSCs group (Fig. 1C, $\mathrm{p}<0.05$ ), although higher relative to UCMSCs 

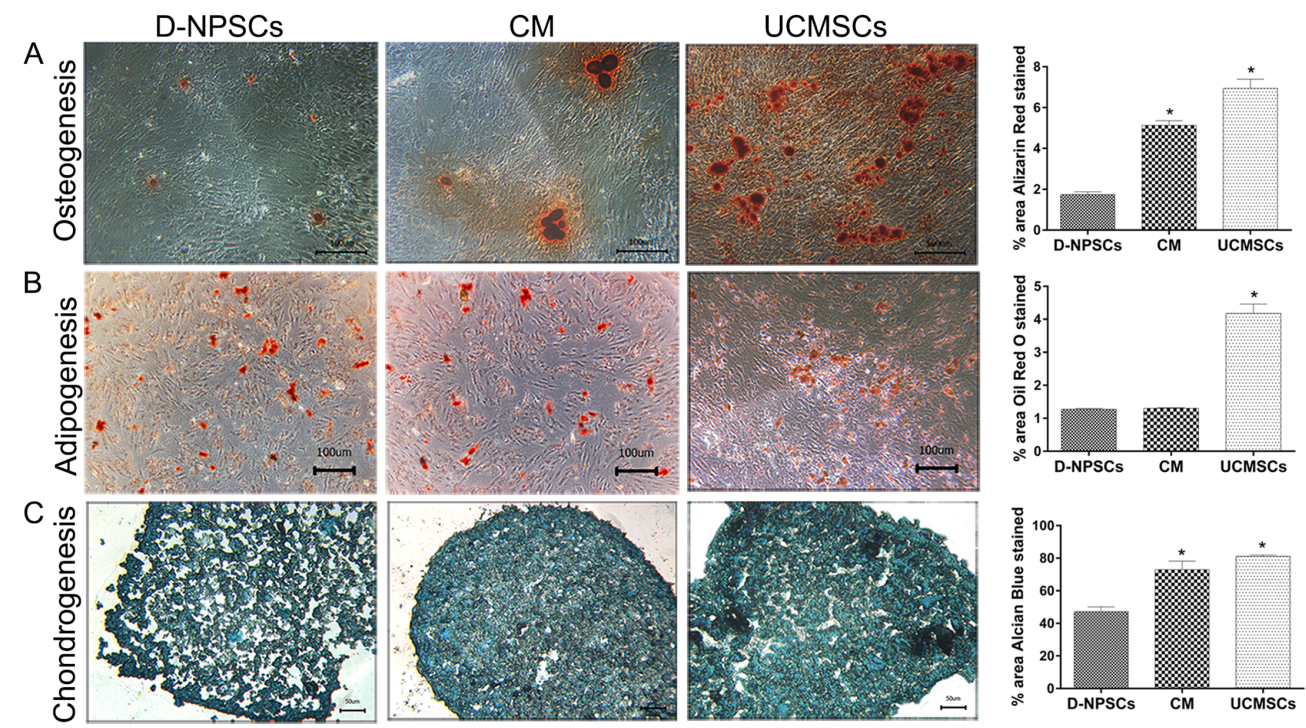

Fig. 5. Multipotent differentiation potential analysis after $72 \mathrm{~h}$ treatment with UCMSCs-CM. (A) Osteogenic differentiation for 21 days,

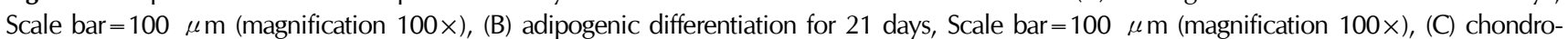
genic differentiation for 21 days, Scale bar $=50 \mu \mathrm{m}$ (magnification $200 \times$ ). The data are presented as the means $\pm S D, n=3$. ${ }^{*} \mathrm{p}<0.01$, vs. D-NPSCs group. D-NPSCs exhibited few calcium deposition stained by Alizarin red S, whereas the cells from CM group displayed larger and more intensely stained mineralized nodules though it presented less intensely staining than UCMSCs. (A) There were no significant difference in Oil red O positive staining area between the CM group and D-NPSCs group, both appeared to form less fat drops than UCMSCs as shown in (B); Cells from CM group produced more intensely stained extracellular matrix than D-NPSCs group, showed similar intensity levels with UCMSCs group. (C) For osteogenic and chondrogenic differentiation, further quantitative analysis also revealed that the percentage of area stained positively was significantly lower in D-NPSCs group than that in both CM group and UCMSCs group.

group, and this result was in accordance with the qRT-
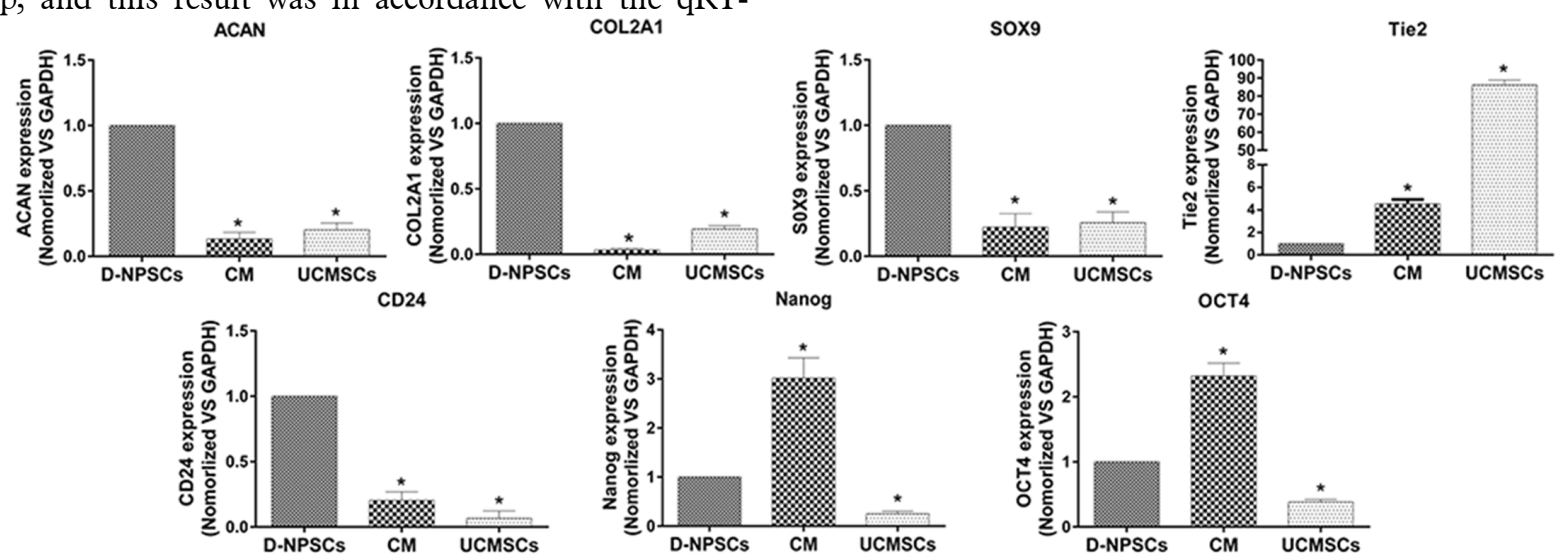

Fig. 6. qRT-PCR for ACAN, COL2A1, SOX9, CD24, Tie2, OCT4 and NANOG was performed after $72 \mathrm{~h}$ treatment with UCMSCs-CM and data were presented as relative ratio to GAPDH. The data are expressed as mean \pm SD. $n=3, * p<0.01$ vs. D-NPSCs. The expression levels of NP cell phenotypic markers, SOX9, COL2A1, ACAN and CD24 in CM group were significantly lower than those in D-NPSCs, while the level of Tie2, OCT4 and NANOG were significantly upregulated in CM group compared to D-NPSCs group.

PCR analysis results.

OCT4 and Nanog were evaluated as pluripotent stem cell markers and the results indicated that the expression levels of these two genes were greatly up-regulated after 72 h UCMSCs-CM treatment (Fig. 6, p $<0.01$ ).

\section{Discussion}

MSCs-based therapies had been proposed as novel treatments for IVD degeneration. Although the initial applications conceived for MSCs therapy were based on their 
multilineage differentiation capacity, subsequent research showed that MSCs engraftment and differentiation at injury sites were very low and transient (28). Increasing evidence indicated that the cytokines and growth factors secreted by MSCs were antiapoptotic, proangiogenic and had the potential to enhance the regenerative capacity of other cells (29-32). Thus, it was hypothesized that MSCs exert their therapeutic effects mainly through secreted trophic factors rather than differentiation. Several studies have shown that MSC-CM can promote the viability and migration of a variety of cells, such as keratinocytes, myocardial cells, endothelial cells and fibroblasts in vitro and in vivo (33-35). However, few studies focused on the therapeutic effects of MSC-CM on degenerated tissue specific progenitor cells.

In the present study, we explored whether MSCs secreted factors can exert beneficial stimulatory effects on D-NPSCs, and characterized changes in immunophenotyping, expression of characteristic NP markers, proliferative capacity and differentiation potentials following treatment of UCMSCs-CM. We observed the expression of MSC specific surface markers CD29 and CD105 was dramatically increased after treating with UCMSCs-CM. Our previous data suggested that NPMSCs from young health tissues expressed all the MSC markers at the same rates as UCMSCs, while the expression of CD29 and CD105 in NPSCs were greatly down-regulated with IVD degeneration. These results indicated that trophic factors in UCMSCs-CM may stimulated D-NPSCs for self-repair, and regained healthier MSCs phenotype.

Similarly, the qRT-PCR results showed that the expression of Tie2 was significantly up-regulated after UCMSCs-CM treatment. Tie2, a cellular membrane receptor tyrosine kinase of the Tie family, was identified recently as a marker of NP precursor cells, which exhibited mulitpotency and self-renewal capacity (36). Notably, the expression of OCT4 and Nanog, the markers of pluripotency were also found greatly up-regulated in D-NPSCs after UCMSCs-CM treatment. Studies have shown that OCT4 and Nanog contributed to the stemness and multi-potency of human MSCs, and knockdown of OCT4 and Nanog in human MSCs reduced cell proliferation rate and differentiation potential $(37,38)$. These results indicated that UCMSCs-CM may improve the stemness and multi-potency of D-NPSCs.

It is noticeable that although the expression of Tie2 significantly up-regulated, while the expression of classic chondrogenic markers, SOX9, COL2A1 and ACAN, and NP cell specific marker CD24 were greatly down-regulated after UCMSCs-CM treatment. Moreover, the ex- pression of OCT4 and Nanog were also significantly up-regulated following UCMSCs-CM treatment. Based on these results, we speculate that UCMSCs-CM improved the undifferentiated state and stemness of D-NPSCs, while reduced the expression of differentiated genes, such as SOX9, COL2A1 and ACAN. UCMSCs-CM may play an important role in dedifferentiation of the D-NPSCs, and consequently increase their multipotency.

Cell growth and proliferation assay fully confirmed that the proliferation capacity of D-NPSCs was remarkably improved by UCMSCs-CM, which was in alignment with the results of cell cycle and apoptosis analysis. As for multilineage differentiation potentials, the results demonstrated that UCMSCs-CM seemed to benefit osteogenesis and chondrogenesis more than adipogenesis in D-NPSCs. The exact underlying mechanism need to be further explored.

Our data demonstrated that UCMSCs-CM improved the stemness, proliferation capacity and potential for osteogenesis and chondrogenesis in D-NPSCs. Previous studies had also revealed that chemokines and growth factors secreted by MSCs were essential for promoting the regenerative capacity of other cells (39). Moreover, Song et al. (40) reported that adipose-derived stem cells improved the viability of nucleus pulposus cells from degenerated IVD in co-culture system. According to above studies, we speculate that CM derived from UCMSCs could promote the regenerative capacity of resident stem/progenitor cells from degenerated disc. The available data demonstrated that IVD degeneration involves not only a declining cell number, but a considerable functional impairment of NP cells. It is therefore evident that enhancing the functionality of NP resident/progenitor cells will be a crucial component in designing future regenerative treatment strategies.

In conclusion, Our finding demonstrated that $\mathrm{CM}$ derived from UCMSCs could promote the regenerative capacity of D-NPSCs by improving their stemness and proliferative capacity, and suggests the possibility of therapeutic strategies to rejuvenate IVD stem/progenitor cells via paracrine signaling, by soluble factors derived from MSCs. This study provides insights and perspectives for the development of a novel therapeutic strategy for IVD degeneration.

\section{Acknowledgments}

This study was supported by the National Natural Science Fund, and Grant number: 81871798, the Special Fund for Scientific Research Fostering of the First Affiliated Hospital, Jinan University, Guangzhou, Guangdong, China, and Grant number 2017207, and the Special 
Fund of Science and Technology of Guangdong Province, and Grant number 2017B030303001.

\section{Potential Conflict of Interest}

The authors have no conflicting financial interest.

\section{Availability of Data and Materials}

All data generated and/or analyzed during this study are included in this published article.

\section{Author Contributions}

Xiaoli Zeng, Jinhua Lin, Jiayue Yu, Hao Wu and Jiaqing Zhang contributed to data collection and study execution, and data analysis and interpretation. Lek Hang Cheang, Mei Tu and Jiaqing Zhang contributed to the study design, data analysis and interpretation, and preparation of the manuscript.

\section{Supplementary Materials}

Supplementary data including one table and one figure can be found with this article online at http://pdf. medrang.co.kr/paper/pdf/IJSC/IJSC-13-s20027.pdf.

\section{References}

1. Sakai D, Andersson GB. Stem cell therapy for intervertebral disc regeneration: obstacles and solutions. Nat Rev Rheumatol 2015;11:243-256

2. Breivik H, Eisenberg E, O'Brien T. The individual and societal burden of chronic pain in Europe: the case for strategic prioritisation and action to improve knowledge and availability of appropriate care. BMC Public Health 2013; 13:1229

3. Wang F, Shi R, Cai F, Wang YT, Wu XT. Stem cell approaches to intervertebral disc regeneration: obstacles from the disc microenvironment. Stem Cells Dev 2015;24:24792495

4. Omlor GW, Fischer J, Kleinschmitt K, Benz K, Holschbach J, Brohm K, Anton M, Guehring T, Richter W. Short-term follow-up of disc cell therapy in a porcine nucleotomy model with an albumin-hyaluronan hydrogel: in vivo and in vitro results of metabolic disc cell activity and implant distribution. Eur Spine J 2014;23:1837-1847

5. Tam V, Rogers I, Chan D, Leung VY, Cheung KM. A comparison of intravenous and intradiscal delivery of multipotential stem cells on the healing of injured intervertebral disk. J Orthop Res 2014;32:819-825

6. Wuertz K, Godburn K, Neidlinger-Wilke C, Urban J, Iatridis JC. Behavior of mesenchymal stem cells in the chemical microenvironment of the intervertebral disc. Spine (Phila Pa 1976) 2008;33:1843-1849
7. Liang C, Li H, Tao Y, Zhou X, Li F, Chen G, Chen Q. Responses of human adipose-derived mesenchymal stem cells to chemical microenvironment of the intervertebral disc. J Transl Med 2012;10:49

8. Johnson WE, Stephan S, Roberts S. The influence of serum, glucose and oxygen on intervertebral disc cell growth in vitro: implications for degenerative disc disease. Arthritis Res Ther 2008;10:R46

9. Erwin WM, Islam D, Eftekarpour E, Inman RD, Karim MZ, Fehlings MG. Intervertebral disc-derived stem cells: implications for regenerative medicine and neural repair. Spine (Phila Pa 1976) 2013;38:211-216

10. Risbud MV, Guttapalli A, Tsai TT, Lee JY, Danielson KG, Vaccaro AR, Albert TJ, Gazit Z, Gazit D, Shapiro IM. Evidence for skeletal progenitor cells in the degenerate human intervertebral disc. Spine (Phila Pa 1976) 2007;32: 2537-2544

11. Mizrahi O, Sheyn D, Tawackoli W, Ben-David S, Su S, Li N, Oh A, Bae H, Gazit D, Gazit Z. Nucleus pulposus degeneration alters properties of resident progenitor cells. Spine J 2013;13:803-814

12. Han B, Wang HC, Li H, Tao YQ, Liang CZ, Li FC, Chen G, Chen QX. Nucleus pulposus mesenchymal stem cells in acidic conditions mimicking degenerative intervertebral discs give better performance than adipose tissue-derived mesenchymal stem cells. Cells Tissues Organs 2014;199: 342-352

13. Li H, Tao Y, Liang C, Han B, Li F, Chen G, Chen Q. Influence of hypoxia in the intervertebral disc on the biological behaviors of rat adipose- and nucleus pulposus-derived mesenchymal stem cells. Cells Tissues Organs 2013;198:266-277

14. Tao YQ, Liang CZ, Li H, Zhang YJ, Li FC, Chen G, Chen QX. Potential of co-culture of nucleus pulposus mesenchymal stem cells and nucleus pulposus cells in hyperosmotic microenvironment for intervertebral disc regeneration. Cell Biol Int 2013;37:826-834

15. Naqvi SM, Buckley CT. Differential response of encapsulated nucleus pulposus and bone marrow stem cells in isolation and coculture in alginate and chitosan hydrogels. Tissue Eng Part A 2015;21:288-299

16. Richardson SM, Hoyland JA. Stem cell regeneration of degenerated intervertebral discs: current status. Curr Pain Headache Rep 2008;12:83-88

17. Wu J, Wang D, Ruan D, He Q, Zhang Y, Wang C, Xin $\mathrm{H}, \mathrm{Xu} \mathrm{C}$, Liu Y. Prolonged expansion of human nucleus pulposus cells expressing human telomerase reverse transcriptase mediated by lentiviral vector. J Orthop Res 2014; 32:159-166

18. Le Maitre CL, Baird P, Freemont AJ, Hoyland JA. An in vitro study investigating the survival and phenotype of mesenchymal stem cells following injection into nucleus pulposus tissue. Arthritis Res Ther 2009;11:R20

19. Saeed H, Ahsan M, Saleem Z, Iqtedar M, Islam M, Danish Z, Khan AM. Mesenchymal stem cells (MSCs) as skeletal therapeutics- an update. J Biomed Sci 2016;23:41 
20. Chen L, Xu Y, Zhao J, Zhang Z, Yang R, Xie J, Liu X, Qi S. Conditioned medium from hypoxic bone marrow-derived mesenchymal stem cells enhances wound healing in mice. PLoS One 2014;9:e96161

21. Hou C, Shen L, Huang Q, Mi J, Wu Y, Yang M, Zeng W, Li L, Chen W, Zhu C. The effect of heme oxygenase-1 complexed with collagen on MSC performance in the treatment of diabetic ischemic ulcer. Biomaterials 2013;34:112-120

22. Sun Z, Liu ZH, Zhao XH, Sun L, Chen YF, Zhang WL, Gao Y, Zhang YZ, Wan ZY, Samartzis D, Wang HQ, Luo ZJ. Impact of direct cell co-cultures on human adipose-derived stromal cells and nucleus pulposus cells. J Orthop Res 2013;31:1804-1813

23. Yamamoto Y, Mochida J, Sakai D, Nakai T, Nishimura K, Kawada H, Hotta T. Upregulation of the viability of nucleus pulposus cells by bone marrow-derived stromal cells: significance of direct cell-to-cell contact in coculture system. Spine (Phila Pa 1976) 2004;29:1508-1514

24. Sakai D, Mochida J, Yamamoto Y, Nomura T, Okuma M, Nishimura K, Nakai T, Ando K, Hotta T. Transplantation of mesenchymal stem cells embedded in Atelocollagen gel to the intervertebral disc: a potential therapeutic model for disc degeneration. Biomaterials 2003;24:3531-3541

25. Wu H, Zeng X, Yu J, Shang Y, Tu M, Cheang LH, Zhang J. Comparison of nucleus pulposus stem/progenitor cells isolated from degenerated intervertebral discs with umbilical cord derived mesenchymal stem cells. Exp Cell Res 2017;361:324-332

26. Wu H, Shang Y, Zhang J, Cheang LH, Zeng X, Tu M. The effects of liquid crystal-based composite substrates on cell functional responses of human umbilical cord-derived mesenchymal stem cells by mechano-regulatory process. J Biomater Appl 2017;32:492-503

27. Richardson SM, Kalamegam G, Pushparaj PN, Matta C, Memic A, Khademhosseini A, Mobasheri R, Poletti FL, Hoyland JA, Mobasheri A. Mesenchymal stem cells in regenerative medicine: focus on articular cartilage and intervertebral disc regeneration. Methods 2016;99:69-80

28. Katsha AM, Ohkouchi S, Xin H, Kanehira M, Sun R, Nukiwa T, Saijo Y. Paracrine factors of multipotent stromal cells ameliorate lung injury in an elastase-induced emphysema model. Mol Ther 2011;19:196-203

29. Rinaldi F, Perlingeiro RC. Stem cells for skeletal muscle regeneration: therapeutic potential and roadblocks. Transl Res 2014;163:409-417

30. Shafei AE, Ali MA, Ghanem HG, Shehata AI, Abdelgawad AA, Handal HR, Talaat KA, Ashaal AE, El-Shal AS. Mesenchymal stem cell therapy: a promising cell-based therapy for treatment of myocardial infarction. J Gene Med 2017;19:e2995

31. Fellows CR, Matta C, Zakany R, Khan IM, Mobasheri A. Adipose, bone marrow and synovial joint-derived mesenchymal stem cells for cartilage repair. Front Genet 2016; 7:213

32. Di Rocco G, Baldari S, Toietta G. Towards therapeutic delivery of extracellular vesicles: strategies for in vivo tracking and biodistribution analysis. Stem Cells Int 2016;2016: 5029619

33. Fong CY, Tam K, Cheyyatraivendran S, Gan SU, Gauthaman K, Armugam A, Jeyaseelan K, Choolani M, Biswas A, Bongso A. Human Wharton's jelly stem cells and its conditioned medium enhance healing of excisional and diabetic wounds. J Cell Biochem 2014;115:290-302

34. Shen C, Lie P, Miao T, Yu M, Lu Q, Feng T, Li J, Zu $\mathrm{T}$, Liu X, Li H. Conditioned medium from umbilical cord mesenchymal stem cells induces migration and angiogenesis. Mol Med Rep 2015;12:20-30

35. Walter MN, Wright KT, Fuller HR, MacNeil S, Johnson WE. Mesenchymal stem cell-conditioned medium accelerates skin wound healing: an in vitro study of fibroblast and keratinocyte scratch assays. Exp Cell Res 2010;316: 1271-1281

36. Sakai D, Nakamura Y, Nakai T, Mishima T, Kato S, Grad S, Alini M, Risbud MV, Chan D, Cheah KS, Yamamura K, Masuda K, Okano H, Ando K, Mochida J. Exhaustion of nucleus pulposus progenitor cells with ageing and degeneration of the intervertebral disc. Nat Commun 2012; 3:1264

37. Seo KW, Lee SR, Bhandari DR, Roh KH, Park SB, So AY, Jung JW, Seo MS, Kang SK, Lee YS, Kang KS. OCT4A contributes to the stemness and multi-potency of human umbilical cord blood-derived multipotent stem cells (hUCB-MSCs). Biochem Biophys Res Commun 2009;384: 120-125

38. Tsai CC, Su PF, Huang YF, Yew TL, Hung SC. Oct4 and Nanog directly regulate Dnmtl to maintain self-renewal and undifferentiated state in mesenchymal stem cells. Mol Cell 2012;47:169-182

39. Li CY, Wu XY, Tong JB, Yang XX, Zhao JL, Zheng QF, Zhao GB, Ma ZJ. Comparative analysis of human mesenchymal stem cells from bone marrow and adipose tissue under xeno-free conditions for cell therapy. Stem Cell Res Ther 2015;6:55

40. Song K, Gu T, Shuang F, Tang J, Ren D, Qin J, Hou S. Adipose-derived stem cells improve the viability of nucleus pulposus cells in degenerated intervertebral discs. Mol Med Rep 2015;12:4664-4668 